\title{
Article \\ Synergistic Enhancement of the Lubrication Performance of Zinc Dialkyldithiophosphate by Coexistence with Ionic Liquid
}

\author{
Kaisei Sato ${ }^{1)^{*}}$, Hikaru Okubo ${ }^{2)}$, Shouhei Kawada ${ }^{3)}$, Seiya Watanabe ${ }^{4)}$ and Shinya Sasaki ${ }^{4)}$ \\ ${ }^{1)}$ Department of Mechanical Engineering, Graduate School of Tokyo University of Science, \\ 6-3-1 Niijuku, Katsushika-ku, Tokyo 125-8585, Japan \\ ${ }^{2)}$ Mechanics and System Engineering, Kyoto Institute of Technology, \\ Gosyokaido-cho, Matsugasaki, Sakyo-ku, Kyoto, Kyoto 606-8585, Japan \\ ${ }^{3)}$ Department of Mechanical Engineering, Kansai University, \\ 3-3-35 Yamate-cho, suita, Osaka 564-8680, Japan \\ ${ }^{4)}$ Department of Mechanical Engineering, Tokyo University of Science, \\ 6-3-1 Niijuku, Katsushika-ku, Tokyo 125-8585, Japan \\ *Corresponding author: Kaisei Sato (4520701@ed.tus.ac.jp)
}

Manuscript received 30 April 2021; accepted 13 August 2021; published 30 September 2021

\begin{abstract}
Ionic liquids (ILs) are being considered as novel lubricant additives to improve the friction and anti-wear characteristics of sliding surfaces. Because ILs consist of only cations and anions, their physical and chemical properties can be easily tailored by modifying their combination. These features enable to design of specific ILs for a given tribological system. However, there are few reports on the effect of ILs used as lubricant additives on the friction and anti-wear characteristics of sliding surfaces. Moreover, it is necessary to investigate the synergism between ILs and other lubricant additives to achieve high practical efficiency. Therefore, in this study, two ILs with different cations but similar anions were used in combination with an anti-wear additive, zinc dialkyldithiophosphate (ZDDP), and the resultant wear and friction characteristics of the worn surfaces were analyzed. We found that the tribological performance under steel/ steel sliding conditions was dependent on the chemical composition of the ILs. In addition, mixed ZDDP and IL solutions exhibited lower friction and wear when compared to ZDDP alone. It is considered that the excellent lubrication and frictional performance observed with the mixed lubricant solutions is due to the formation of tribofilms comprising of ZDDP and IL on the sliding surface.
\end{abstract}

\section{Keywords}

ionic liquid, zinc dialkyldithiophosphate, lubricant additive, tribofilm, surface analysis

\section{Introduction}

Recent environmental issues across the world have highlighted the importance of improving automobile fuel efficiency [1-6]. The use of lower viscosity oils to reduce fluid resistance under hydrodynamic lubrication is one way to improve fuel efficiency in automotive engines [4]. However, the lower viscosity of these oils may result in seizure and/ or increase wear under boundary and mixed lubrication conditions. Therefore, it is necessary to study and optimize lubricants to improve the performance and durability of sliding machine elements [6]

In such a background, anti-wear additives play essential roles in improving and extending the lifespan of engine components. One of the typical anti-wear additives: zinc dialkyldithiophosphate (ZDDP), which is a multifunctional additive indispensable for lubricating oil due to its anti- oxidation, anti-seizure, and anti-wear properties, has been used since the 1940s [7-13]. Excellent deal research has already reported three main mechanisms of the anti-wear action on steel: (i) a reduction effect of adhesion wear by forming a mechanically protective film [8], (ii) a removal effect of corrosive peroxides or peroxy radicals $[9,10]$ and (iii) a digesting effect of hard and thus abrasive iron oxide particles [11, 12]. Under boundary lubrication, anti-wear additives form a tribofilm during rubbing to protect the surface from adhesive wear by preventing direct metal/metal contacts [7]. ZDDP also forms rough, patchy, and pad-like films with a thickness of about $100-150 \mathrm{~nm}$ and a width of about 2-6 $\mu \mathrm{m}[7,8,13]$. The ZDDP tribofilms are composed of three layers: a bottom layer of iron and zinc sulfide, a middle layer of zinc and iron phosphates/polyphosphate, and a top layer of mixed oxide/zinc sulfide layer [7, 14]. In the initial formation of tribofilm, iron and zinc sulfides are produced on the rubbing steel surface [15], and this has a protective effect at extreme 
pressure [16]. In the subsequent formation process, which is under mild rubbing conditions, zinc phosphate/polyphosphatebased tribofilms mainly form on top of these sulfides with mixing it [15]. It has also been suggested that the anti-wear film behaves like a cushion, reducing the stress caused by substrate asperity peaks [17] and these layers. However, there are severe issues using ZDDPs that cause catalyst poisoning, generating sludge, and corroding copper-based alloy components. Therefore, the combined use of ZDDP and other additives is expected to reduce ZDDP concentration and improve friction and anti-wear properties in lubricants [18-20].

Ionic liquids (ILs) are salts composed of cations and anions in the liquid state. Recently, ILs are being considered for use as new lubricant additives owing to their excellent characteristics such as low vapor pressure, high thermal stability, high ionic conductivity, and high chemical stability [21]. Because they are composed of only cations and anions, their chemical structure and resultant properties can be easily controlled [21]; these features make it possible to design IL for a particular tribological systems. In addition, ILs have exhibited the promising potential to improve tribological properties as lubricant usage [2226]. Especially, fluorine-based ILs are frequently utilized as lubricants [26]. These ILs exhibit a low friction coefficient due to the formation of the tribofilms consisting of metallic fluoride [22, $27,28]$.

Effective anti-wear and friction-reduction functionalities have been observed when adding ILs into lubricating oils. Qu et al. reported that when a phosphonium-based IL was used as an additive, anti-wear IL-derived tribofilms were formed on sliding surfaces, which led to a reduction in friction and wear $[18-20,25]$. Also, the anti-wear and frictional properties are superior to ZDDP containing oil [18-20, 26]. In addition, from these reports, it is believed that the ILs tribofilms have a layered structure and cause the low shear strength by preventing direct contact between two rubbing substances while ZDDP form regid tribofilms [18-20, 26, 29]. On the other hunds, Cai et al. reported that the frictional properties were improved by increasing the additive amount of imidazolium IL from 0.5 to 5.0 mass \% while the lowest wear volume was shown when adding 1.0 mass $\%$ of ILs [30, 31]. However, commercial engine lubricants contain several categories of additives such as anti-wear, friction modifier, viscosity modifier, anti-oxidant, detergent, and dispersant additives $[7,8]$. Therefore, it is essential to understand the synergistic effect of ILs and other additives on friction and anti-wear properties by changing the chemical structure of ILs to expand their application.

From these backgrounds, we focused on the combined use of ILs and ZDDP to improve the friction and anti-wear propeties while reducing the ZDDP concentration. Recently, $\mathrm{Qu}$ et al. examined friction and anti-wear properties on the combined use of ZDDP and phosphonium ILs and confirmed synergistic effects between ZDDP and the ILs [32, 33]. In their reports, chemistry and bonding states for each tribofilm were examined in detail by atom probe tomography (APT), scanning transmission electron microscopy (STEM) imaging, and electron energy loss spectroscopy (EELS). They indicated that the composition of the tribofilm formed by ZDDP and IL solution is largely different from that of ZDDP alone solution [32, 33]. In addition, they proposed an anion exchange hypothesis that a new compound, which is a zinc alkylphosphate alkyldithiophosphate (ZOTP) composed of ZDDP and IL, is generated in ZDDP + IL mixed solution and the ZOTP improves the friction and anti-wear properties [32]. However, the relationship between the chemical structure of ILs and the tribological properties of the tribofilm formed by ZDDP and ILs solution has yet to be clarified.

In this study, the purpose is to investigate the synergistic effect of ILs and ZDDP when using two types of ILs with different chemical structures. Mainly, we focused on the effect of physical properties on tribofilm formation by ZDDP and ILs and investigated the friction and anti-wear properties using a reciprocating sliding tester. In order to make the contrast between the ILs, we used the ILs with the same anion and different two cations. The friction and anti-wear characteristics of the IL and ZDDP mixed solutions were investigated using a reciprocating-type tribotester and the lubrication mechanism was elucidated by atomic force microscopy (AFM), time-offlight secondary ion mass spectrometry (ToF-SIMS), and X-ray photoelectron spectroscopy (XPS).

\section{Experimental methods}

\subsection{Materials and lubricants}

A bearing steel disk $(\phi=24 \mathrm{~mm}$ and $\mathrm{t}=7.9 \mathrm{~mm}$, AISI 52100 with a hardness of $700 \mathrm{HV}$ and surface roughness (Ra of 0.01 $\mu \mathrm{m})$ and bearing steel cylinder $(\phi=15 \mathrm{~mm}$ and $1=22 \mathrm{~mm}$, AISI 52100 with a hardness of $700 \mathrm{HV}$ and Ra of $0.04 \mu \mathrm{m}$ ) were used for friction tests.

We used dioctyl sebacate (DOS) with a viscosity of 18 $\mathrm{mPa} \cdot \mathrm{s}$ at $25^{\circ} \mathrm{C}$ as the base oil. DOS was procured from Sanwa Kasei Kogyo Co., Japan. The selection of base oils is important for incorporating ILs as additives as most ILs exhibit minimal solubility in non-polar hydrocarbon oils. Thus, DOS, which is an ester-based oil is suitable because of its thermal stability and good additive solubility [34,35]. Sanes et al. reported that esterbased oils are more suitable as solvents for ILs when compared to mineral oils or poly-alpha-olefins $[24,36]$.

In addition to ZDDP (alkyl-structure: secondary C-3 \& C-6 mixed; ADEKA Co., Tokyo, Japan), two ILs, viz. 1-butyl-1methylpyrrolidinium tris(pentafluoroethyl) trifluorophosphate ([BMPL][FAP]) and 1-butyl-3-methylimidazolium tris(pentafluoroethyl) trifluorophosphate ([BMIM][FAP]) were used in this study. The two ILs were procured from Merck Co., Germany ( $>99 \%$ purity and water content $<100 \mathrm{ppm}$ ). These two ILs, which contained the same [FAP] anion, were selected because they were reported to be soluble in ester oils [37]. In order to design ILs with selective tribological properties, it is necessary to understand the relationship between their chemical structures and friction and anti-wear properties; thus, we chose [BMPL][FAP] and [BMIM][FAP], which contained the same anion but different cations, as ILs. By comparing an imidazolium-based and a pyrrolidinium-based ionic ILs having the same alkyl chain length, we investigated the effect of physical properties induced by the difference in cation structure on the friction properties. The viscosities of [BMPL][FAP] and [BMIM][FAP] were 292 and $93 \mathrm{mPa} \cdot \mathrm{s}$, respectively. Table 1 shows the chemical structures of DOS, ZDDP, [BMIM][FAP], and [BMPL][FAP].

The lubricant combinations used were DOS, DOS containing 0.67 mass\% ZDDP (DOS + ZDDP), DOS containing 1.5 mass\% of each IL (DOS + [BMPL][FAP] and DOS + [BMIM][FAP]), and DOS containing 0.465 mass $\%$ of ZDDP and 0.465 mass $\%$ of the ILs (DOS + ZDDP + [BMPL][FAP] and DOS + ZDDP + [BMIM] [FAP]). All the additive-containing lubricants were adjusted to a phosphorus concentration of $800 \mathrm{ppm}$ to apply for engine oil, which concentration is the limit of engine oil limitation [38]. 
Table 1 Chemical structures of the base oil and additives

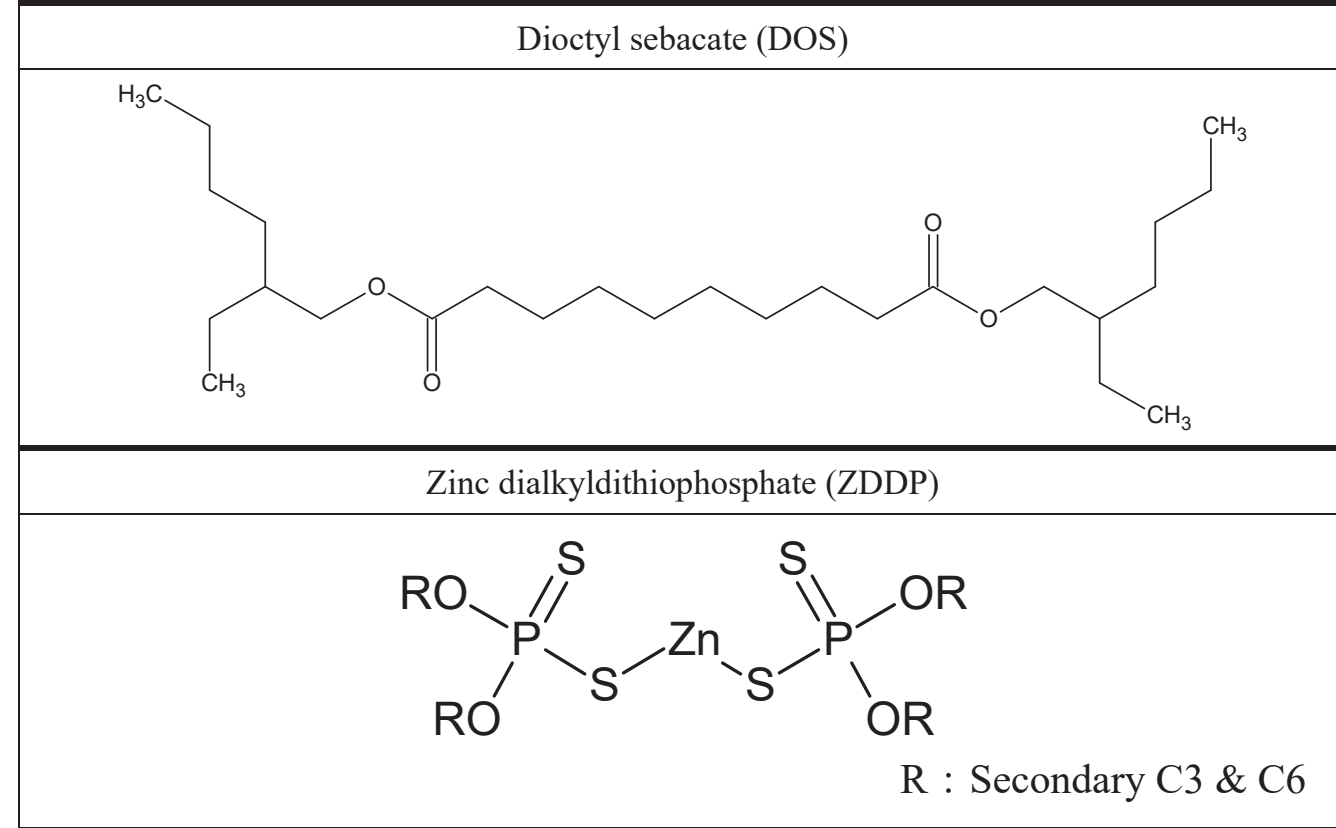

1-butyl-1-methylpyrrolidinium tris(pentafluoroethyl) trifluorophosphate ([BMPL][FAP])

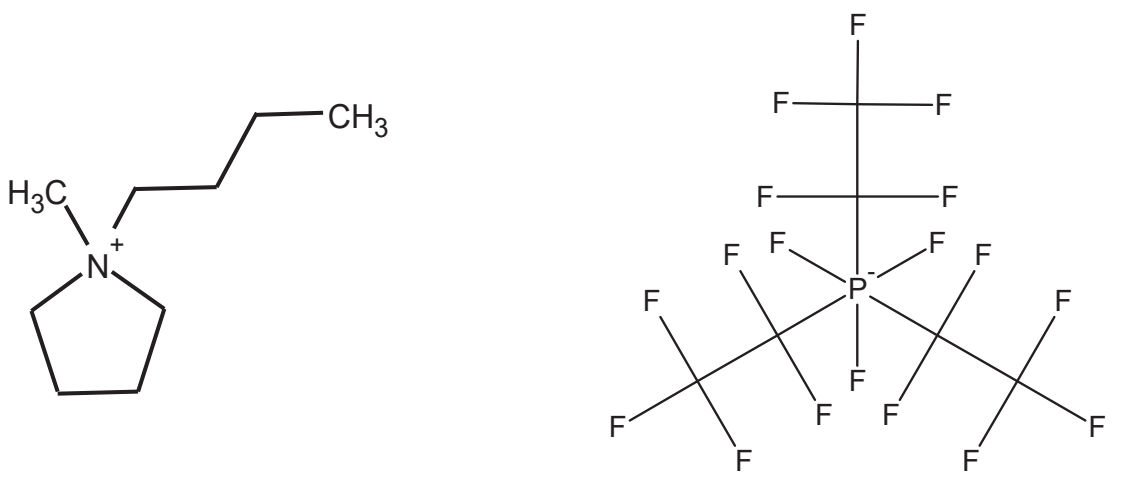

1-butyl-3-methylimidazolium tris(pentafluoroethyl) trifluorophosphate ([BMIM][FAP])

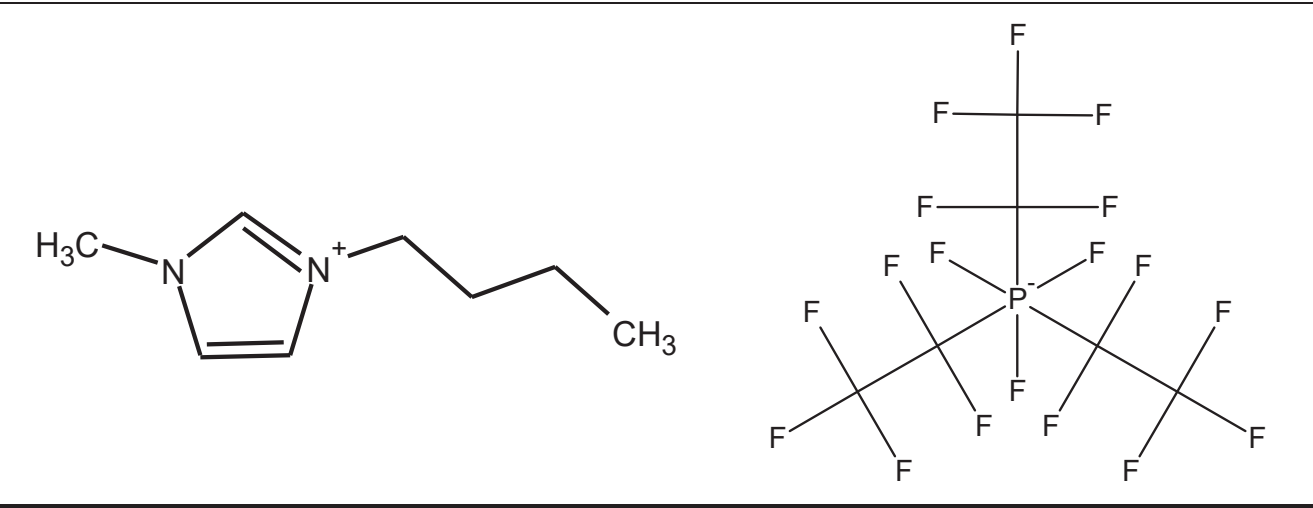

\subsection{Sliding test}

Tribological properties were evaluated using a reciprocating-type cylinder-on-disk tribotester (SRV4, Optimol, Germany). Figure 1 shows a schematic representation of the SRV tribotester. Friction tests were conducted for $60 \mathrm{~min}$ on a steel/ steel tribo-pair at a load of $200 \mathrm{~N}$ (corresponding to a maximum initial Hertzian contact pressure of $250 \mathrm{MPa}$ ), cylinder stroke of $1.0 \mathrm{~mm}$, frequency of $50 \mathrm{~Hz}$, and temperature of $80^{\circ} \mathrm{C}$; $90 \mu \mathrm{L}$ of the lubricant was applied on the sliding surfaces before testing. The test condition was set for the lambda ratio to be lower than 1.0 , and it was calculated to be 0.52 . The system is considered to be at the boundary lubrication condition during the sliding test. Each wear volume was calculated from the width of the wear tracks on the cylinder, which were evaluated by confocal laser scanning microscopy (VK-X150, KEYENCE, Japan). 
2.3 Thermal stability of ILs

The thermal stabilities of the ILs were evaluated by thermogravimetric analysis (TGA; TG-DTA2010SA, Bruker, USA). The sample and reference pans were made of aluminum and measurements were carried out in an inert $\mathrm{N}_{2}$ environment. The temperature programming rate was set at $10^{\circ} \mathrm{C} \min ^{-1}$ and tests were conducted in the range of $60-500^{\circ} \mathrm{C} . \mathrm{Al}_{2} \mathrm{O}_{3}$ was used as the reference material. The combined mass of the IL and reference materials was $\sim 10 \mathrm{mg}$.

\subsection{Surface analysis of wear tracks}

Surface analysis was carried out on each wear track on each disk specimen using AFM (S-image, Hitachi High-Tech Corporation, Japan), ToF-SIMS (ULVAC TRIFT III, ULVAC PHI, Inc., Japan), and XPS (AXIS Nova, Kratos Analytical, UK, AlK $\alpha$ X-ray source, scan area with diameter $\phi=100 \mu \mathrm{m})$. The test specimens before surface analysis were cleaned by slowly shaking them in $50 \mathrm{ml}$ of $\mathrm{n}$-hexane for 30 seconds.

\subsubsection{AFM analysis}

AFM was conducted in the contact mode to investigate specimen surface characteristics after sliding tests; the observed topographies $\left(10 \times 10 \mu \mathrm{m}^{2}\right)$ can be used to determine the structure of the composites [7, 8, 13, 39, 40]. A silicon probe (SI-DF20, Hitachi High-Tech, Japan) with a nominal tip radius of $10 \mathrm{~nm}$ and a spring constant of $14 \mathrm{~N} / \mathrm{m}$ was used for AFM measurements. Force-displacement (FD) curves were obtained by nanoindentation using AFM and these curves yield information on the nano-physical properties of the tested surfaces [41-46].

\subsubsection{ToF-SIMS analysis}

A pulsed electron impact ion source $(15 \mathrm{keV}, 69 \mathrm{Ga}+)$ was used to generate primary ions for the analysis. The analysis area on the sample was $100 \mu \mathrm{m} \times 100 \mu \mathrm{m}$. Ion irradiation time was 5 min. Mass resolution was $500 \mathrm{at} \mathrm{m} / \mathrm{z}=27(\mathrm{Al})$. Lateral resolution was $2 \mu \mathrm{m}$.

\subsubsection{XPS depth analysis}

XPS survey spectra were recorded from $-10 \mathrm{eV}$ to $1300 \mathrm{eV}$ with a pass energy of $160 \mathrm{eV}$. Narrow scans were performed on 8 chemical elements of $\mathrm{Zn} 2 \mathrm{p}, \mathrm{Fe} 2 \mathrm{p}, \mathrm{O} 1 \mathrm{~s}, \mathrm{~N} 1 \mathrm{~s}, \mathrm{C} 1 \mathrm{~s}, \mathrm{~F}$ 1s, $\mathrm{S} 2 \mathrm{p}$, and P $2 p$ with a pass energy of $40 \mathrm{eV}$ and the number of scans was set to 16 for Zn $2 p$ and Fe 2p, and 4 for O 1s, N 1s, C 1s, F 1s, S 2p, and P 2p. Each peak was calibrated with reference to the binding energy (BE) of $\mathrm{C} 1 \mathrm{~s}$ at $284.8 \mathrm{eV}$. To confirm the chemical composition of the tribofilms formed by DOS + ZDDP, $\mathrm{DOS}+\mathrm{ZDDP}+[\mathrm{BMPL}][\mathrm{FAP}]$, and DOS + ZDDP + [BMIM] [FAP], XPS depth analysis with Ar ion etching using an ion gun (beam energy, $4 \mathrm{keV}$; diameter, $1 \mathrm{~mm}$ ) was carried out on

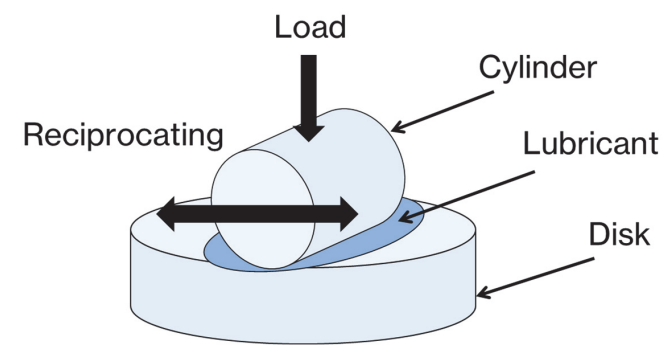

Fig. 1 Schematic of the sliding tester each wear track on the disk specimen $[47,48]$. This analysis enables to evaluate tribofilm in a depth direction, hence useful to understand the detailed chemical structure of tribofilm. However, it has also been reported that chemical shifts may occur due to sputtering $[49,50]$. In this experiment, we used steel specimens. J.B. Malherbe et al. reported that the chemical shifts of iron oxide and zinc oxide are small by ion sputtering [51]. Therefore, we consider that the influence of Ar sputtering on iron composites and zinc composites is negligible.

\section{Results}

\subsection{Friction and wear properties}

Figure 2 illustrates the frictional behavior of each lubricant solution as a function of sliding time. In the case of DOS, DOS + [BMPL][FAP], and DOS + [BMIM][FAP] solutions, the observed friction behavior was unstable throughout the test; furthermore, the friction coefficients obtained with DOS + [BMPL][FAP] and $\mathrm{DOS}+[\mathrm{BMIM}]$ were higher than that obtained with pure DOS. Meanwhile, the friction coefficients obtained with DOS + ZDDP, $\mathrm{DOS}+\mathrm{ZDDP}+[\mathrm{BMPL}][\mathrm{FAP}]$, and DOS + ZDDP + [BMIM][FAP] decreased gradually and became constant. It is to be noted that these values were lower than those obtained with pure DOS.

Figure 3 shows the average friction coefficient obtained with each lubricant. Each friction test was conducted three times and the obtained values were averaged. The average friction coefficients obtained with DOS, DOS + [BMPL][FAP], and DOS + [BMIM] [FAP] were $0.155,0.155$, and 0.167 , respectively.

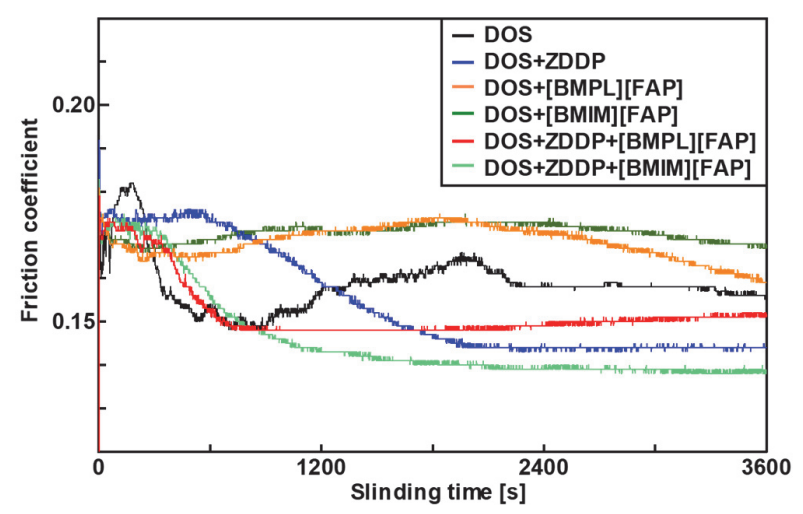

Fig. 2 Friction behavior of different lubricant systems

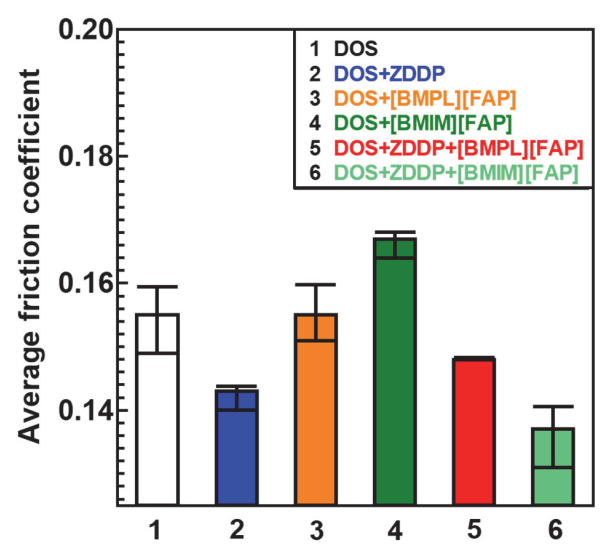

Fig. 3 Average friction coefficients obtained with different lubricant systems 
Meanwhile, the average friction coefficients obtained with DOS + ZDDP, DOS + ZDDP + [BMPL][FAP], and DOS + ZDDP + [BMIM][FAP] were $0.143,0.148$, and 0.137 , respectively. These values were lower than those of pure DOS and while DOS + ZDDP + [BMIM][FAP] resulted in a smaller friction coefficient than DOS + ZDDP, DOS + ZDDP + [BMPL][FAP] resulted in a higher friction coefficient. These results show that changing the cation component in the IL (and thus its structure) resulted in different friction characteristics in ZDDP + IL mixed solutions.

Figure 4 shows the average wear volume (from three repeated tests) of steel cylinders lubricated with different systems at the end of friction testing. Compared to pure DOS, all other lubricants resulted in higher wear reduction. The wear volume observed with all ZDDP-containing solutions was smaller than that with pure DOS by $\sim 60 \%$. In particular, DOS+ ZDDP + [BMIM][FAP] showed the lowest wear volume, followed by DOS + ZDDP + [BMPL][FAP], and DOS + ZDDP DOS + ZDDP + [BMIM] [FAP] resulted in a significant wear reduction of $35 \%$ when compared to DOS+ ZDDP.

\subsection{Thermal stability of ILs}

The thermal stabilities of the ILs were evaluated by TGA; Figure 5 shows their weight-loss behavior with respect to the heating temperature. As shown in the figure, the decomposition temperatures of [BMPL][FAP] and [BMIM][FAP], defined as

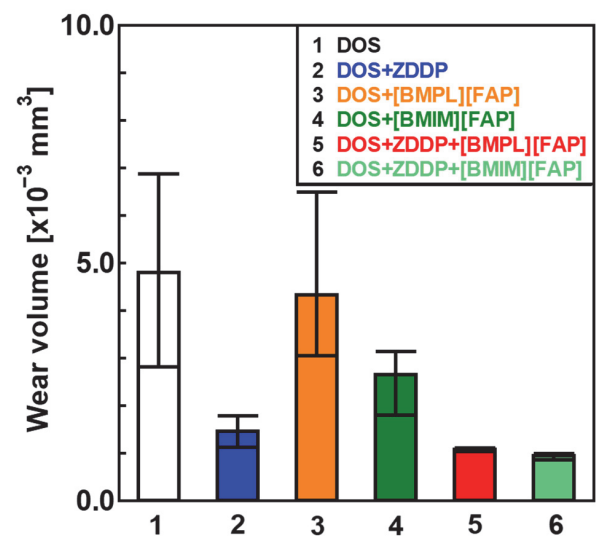

Fig. 4 Average wear volumes obtained with different lubricant systems

(a)

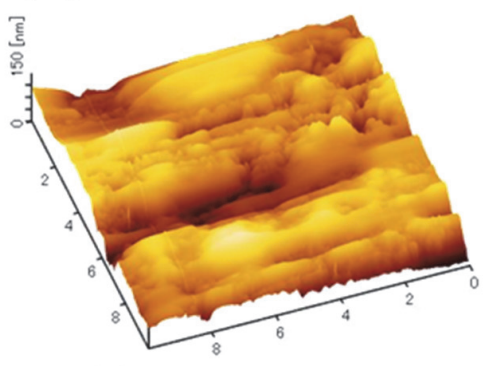

[um] the temperature of $5 \%$ weight loss, were 346.9 and $341.5^{\circ} \mathrm{C}$, respectively.

\subsection{Surface analysis of wear tracks}

3.3.1 AFM analysis

Figure 6 shows the topographies of worn surfaces lubricated with DOS + [BMPL][FAP], and DOS + [BMIM][FAP]. Figure 7 shows the topographies of worn surfaces lubricated with DOS + ZDDP, DOS + ZDDP + [BMPL][FAP], and DOS + ZDDP + [BMIM][FAP]. In the case of lubricants containing IL alone, the worn surfaces were the shape such as wear scar along with the sliding direction. On the other hand, in the case of lubricants containing ZDDP, the shapes were patchy and rough. These results suggested that pad-like structures were formed on the worn surfaces with each solution [7, 8, 13]. Feature heights in the AFM images of surfaces lubricated with DOS + ZDDP, DOS $+\mathrm{ZDDP}+[\mathrm{BMPL}][\mathrm{FAP}]$, and DOS + ZDDP + [BMIM][FAP] were $\sim 150,120$, and $120 \mathrm{~nm}$, respectively. These results suggest that ZDDP formed the pad-like structures in the case of lubricants containing ZDDP.

Figure 8 shows the FD curves of worn surfaces lubricated with DOS + ZDDP, DOS + ZDDP + [BMPL][FAP], and DOS + ZDDP + [BMIM][FAP]. These results show that DOS + ZDDP + IL solutions formed highly viscous films on the outermost surfaces. In the case of the DOS + ZDDP lubricant (Fig. 8(a)),

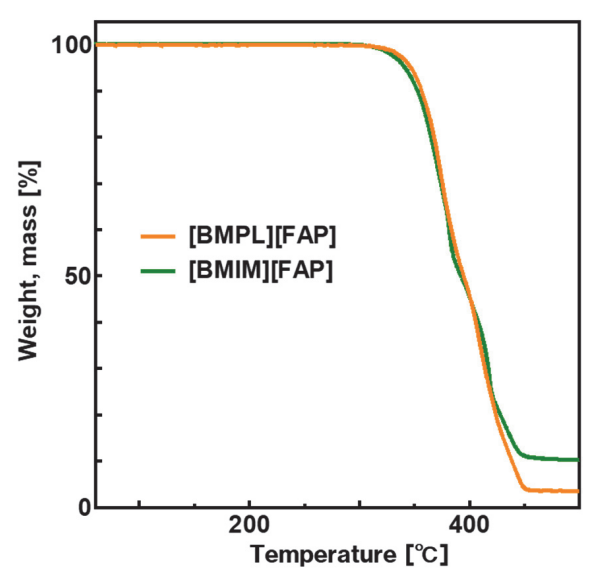

Fig. 5 TGA thermograms of [BMPL][FAP] and [BMIM][FAP]

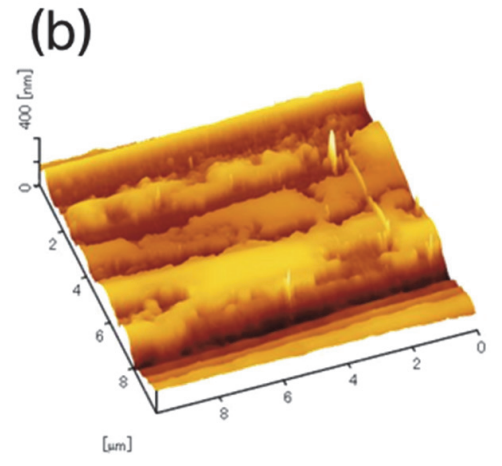

Fig. 6 3D AFM topographies of sliding surfaces lubricated with (a) DOS + [BMPL][FAP], and (b) DOS + [BMIM][FAP] 
(a)

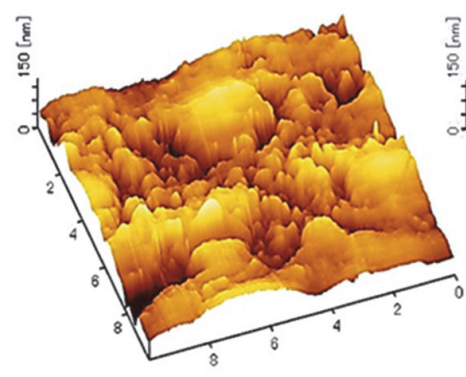

[um] (b)

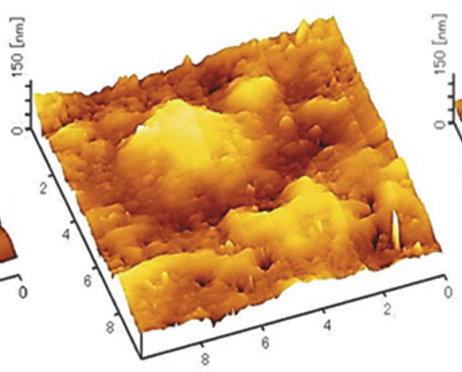

$[\mathrm{mm}]$

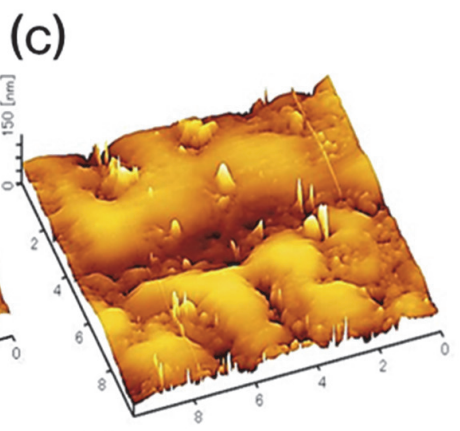

[mm]

Fig. 7 3D AFM topographies of sliding surfaces lubricated with (a) DOS + ZDDP, (b) DOS + ZDDP + [BMPL][FAP], and (c) $\mathrm{DOS}+\mathrm{ZDDP}+[\mathrm{BMIM}][\mathrm{FAP}]$

(a)

(b)

(c)

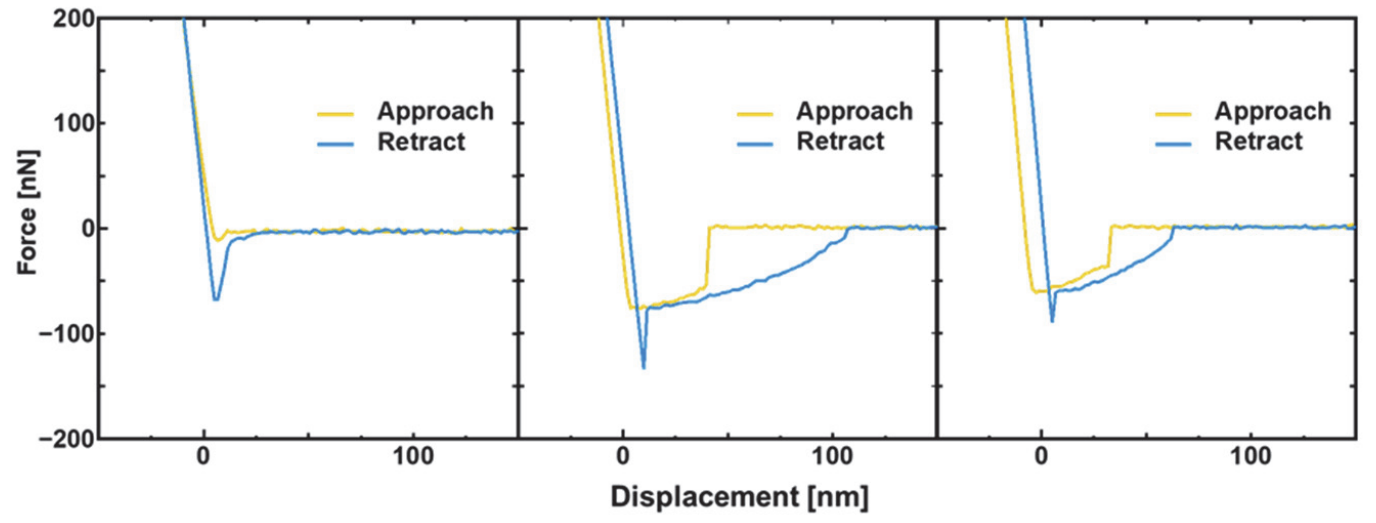

Fig. 8 FD curves obtained with (a) DOS + ZDDP, (b) DOS + ZDDP + [BMPL][FAP], and (c) DOS + ZDDP + [BMIM][FAP]

the cantilever was suddenly attracted by an adhesive force when it approached the surface. In the retraction process, the cantilever was suddenly retracted from the surface. Meanwhile, in the case of DOS + ZDDP + IL solutions (Fig. 8(b) and (c)), the cantilever was attracted during the approach process owing to long-range attractive interactions, which gradually increased. In the retraction process, the cantilever did not instantly retract from the surface, unlike the phenomenon observed with DOS + ZDDP. Instead, the adhesive force gradually decreased as the piezo distance increased. This indicates the existence of a layer with high viscosity. In the case of DOS + ZDDP + IL solutions, highly viscous layer was formed on the outermost surface of the worn specimen.

\subsubsection{ToF-SIMS analysis}

To investigate the chemical composition of the outermost surface (depth of 2-3 nm) of worn specimens lubricated with different solutions, ToF-SIMS was conducted [21, 52-58]. Figures 9 and 10 show the positive and negative spectra obtained from the worn surfaces lubricated with DOS + ZDDP, DOS + ZDDP $+[\mathrm{BMPL}][\mathrm{FAP}]$, and DOS + ZDDP + [BMIM][FAP]. In all the positive spectra, $\mathrm{Zn}^{+}$, which were derived from $\mathrm{ZDDP}$, could be detected. In the negative spectra, peaks corresponding to $\mathrm{S}$ - could be observed in addition to those corresponding to phosphoric acid [7, 8]. This implies that a ZDDP tribofilm was formed on the worn surfaces lubricated with DOS + ZDDP, $\mathrm{DOS}+\mathrm{ZDDP}+[\mathrm{BMPL}][\mathrm{FAP}]$, and DOS + ZDDP + [BMIM][FAP].
In addition, peaks corresponding to $[\mathrm{BMPL}]^{+}$and $[\mathrm{BMIM}]^{+}$were detected in the positive spectra of DOS + ZDDP + [BMPL][FAP] and DOS + ZDDP + [BMIM] [FAP], respectively. F', C2F5', and $[\mathrm{FAP}]^{-}$were also detected in the negative spectra of the ZDDP + IL solutions, which indicates that mixed tribofilms were formed on the test surfaces.

To analyze the chemical composition at the outermost surface, the abundance ratios of ILs and ZDDP compounds were compared on the worn surfaces. The count ratio, which is defined as the ratio of the peak counts of each ion and the total ion counts, was calculated as shown in Fig. $11\left(\mathrm{Zn}^{+}\right.$counts/ total counts (positive ion), $\mathrm{S}^{-}$counts/total counts (negative ion), $[\mathrm{BMIM}]^{+}$or $[\mathrm{BMPL}]^{+}$counts/total counts (positive ion), and $\left.{ }^{[F A P]}\right]^{-}$counts/total counts (negative ion)). These results show that more ILs were detected than ZDDP-derived elements. This indicates that the pure ILs exist at the outermost surface of the tribofilms derived from DOS + ZDDP + [BMPL][FAP] and DOS + ZDDP + [BMIM] [FAP].

\subsubsection{XPS analysis of the tribofilm surface}

The amount of ZDDP-derived and IL-derived compounds was estimated from the intensity area ratio of each XPS spectrum and the sensitivity factor corresponding to each peak area. Figures 12 and 13 map the abundance of Fe 2p, O 1s, C 1s, and $\mathrm{N}$ 1s and $\mathrm{P} 2 \mathrm{p}, \mathrm{S} 2 \mathrm{p}$, and $\mathrm{Zn} 2 \mathrm{p}$ as functions of the etching time, respectively. As shown in Fig. 12, as a function of etching time, the abundance of Fe $2 p$ increases, and the abundance of 
$\left[\times 10^{+3}\right]$

(a)

(b)

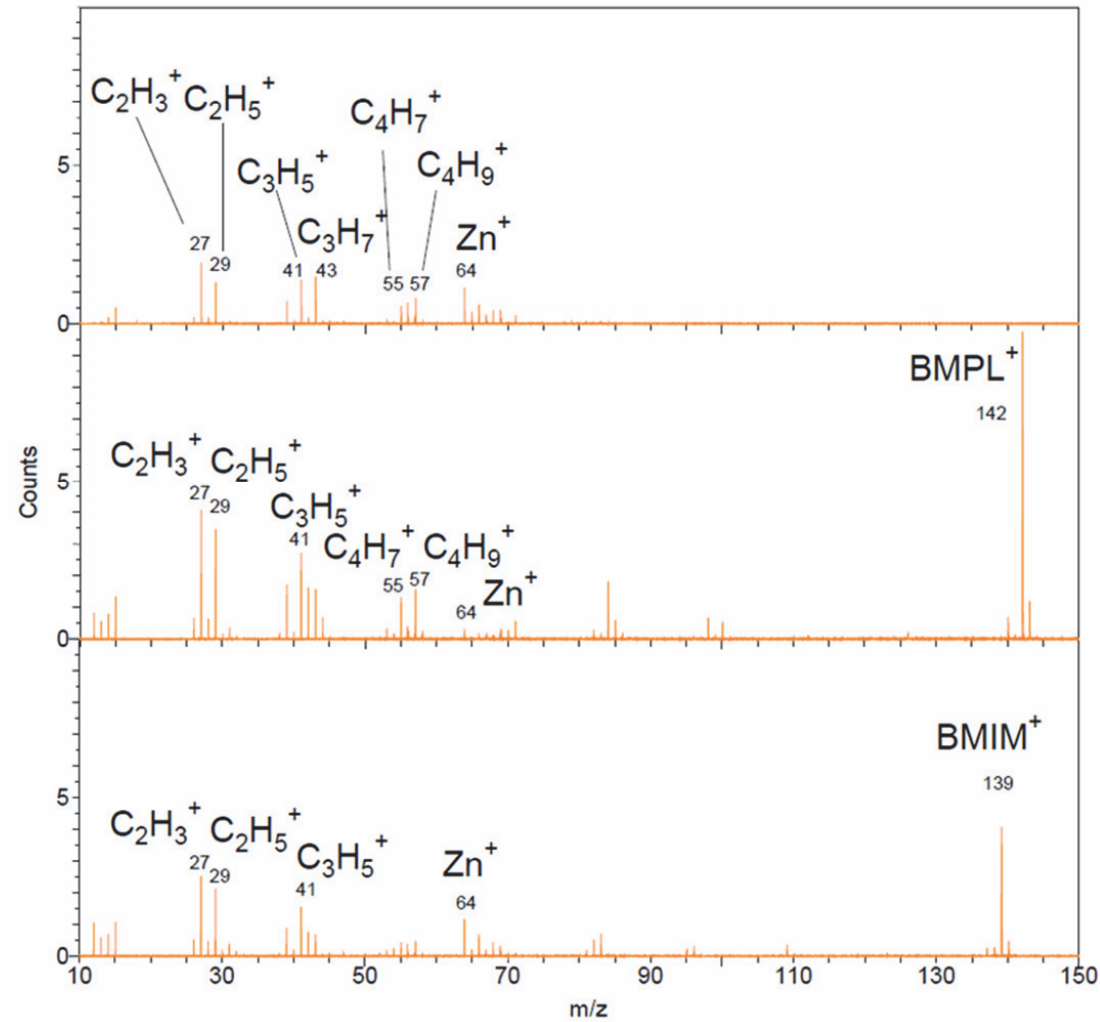

Fig. 9 ToF-SIMS positive spectra of worn surfaces lubricated with (a) DOS + ZDDP, (b) DOS + ZDDP + [BMPL][FAP], and (c) DOS + ZDDP + [BMIM][FAP]

$\left[\times 10^{+4}\right]$

(a)

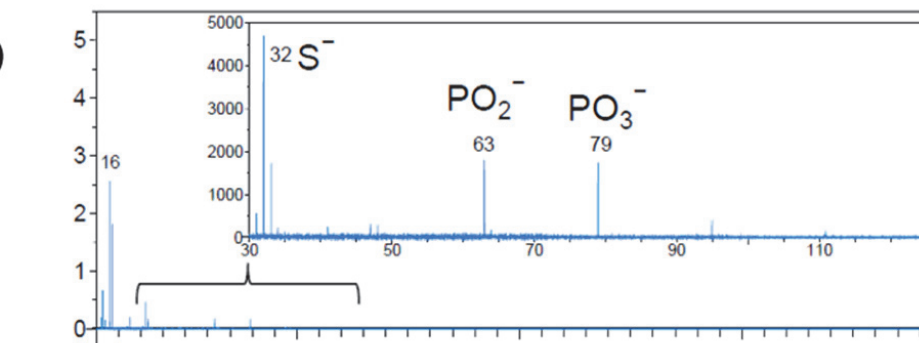

(b)

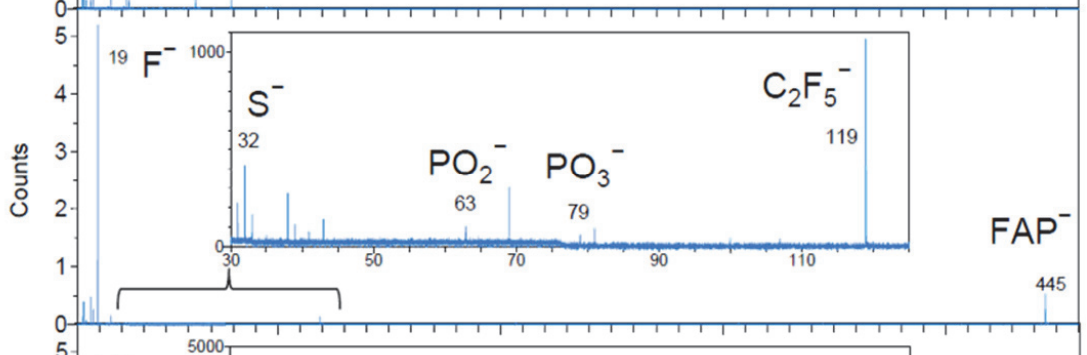

(c)

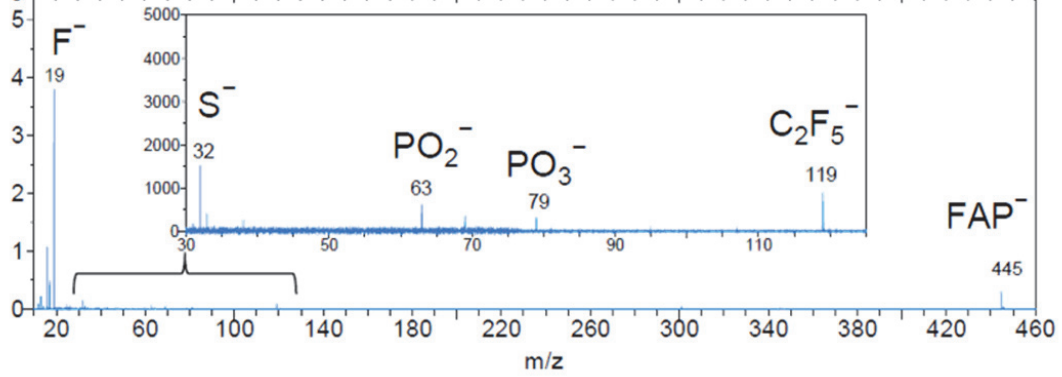

Fig. 10 ToF-SIMS negative spectra of worn surfaces lubricated with (a) DOS + ZDDP, (b) DOS + ZDDP + [BMPL][FAP], and (c) DOS + ZDDP + [BMIM] [FAP] 
(a) DOS + ZDDP + [BMPL][FAP]
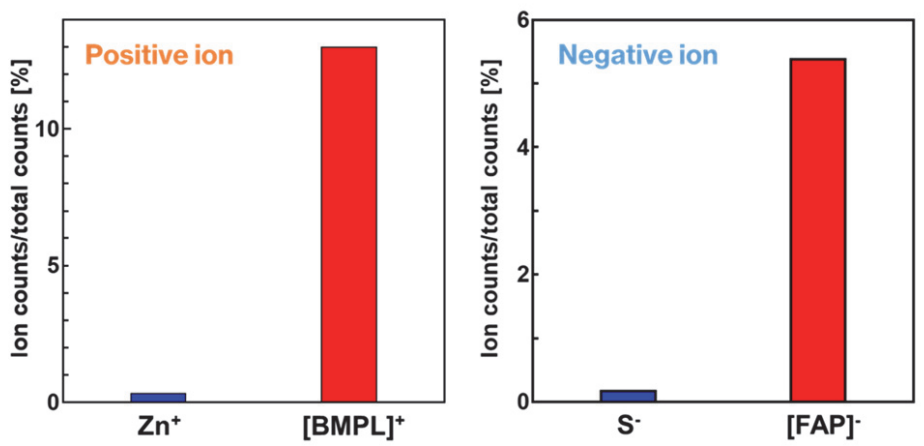

(b) DOS + ZDDP + [BMIM][FAP]
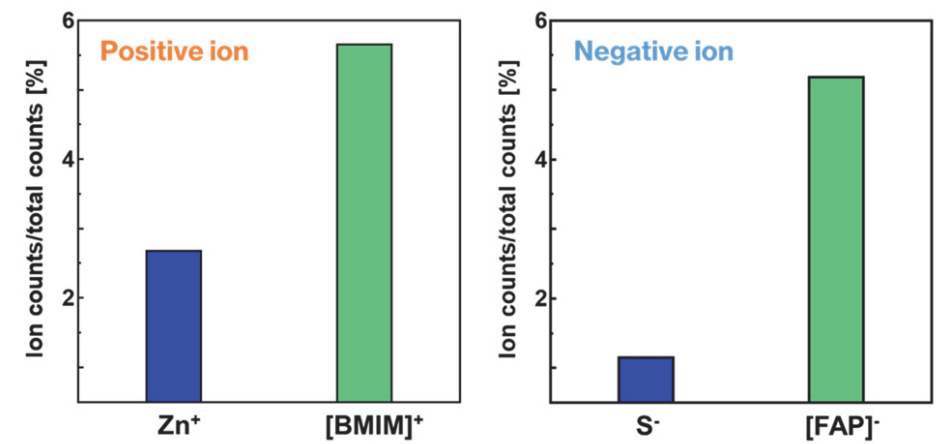

Fig. 11 Count ratio at the outermost surfaces of worn substrates lubricated with (a) DOS + ZDDP + [BMPL][FAP], and (b) DOS + ZDDP + [BMIM][FAP]
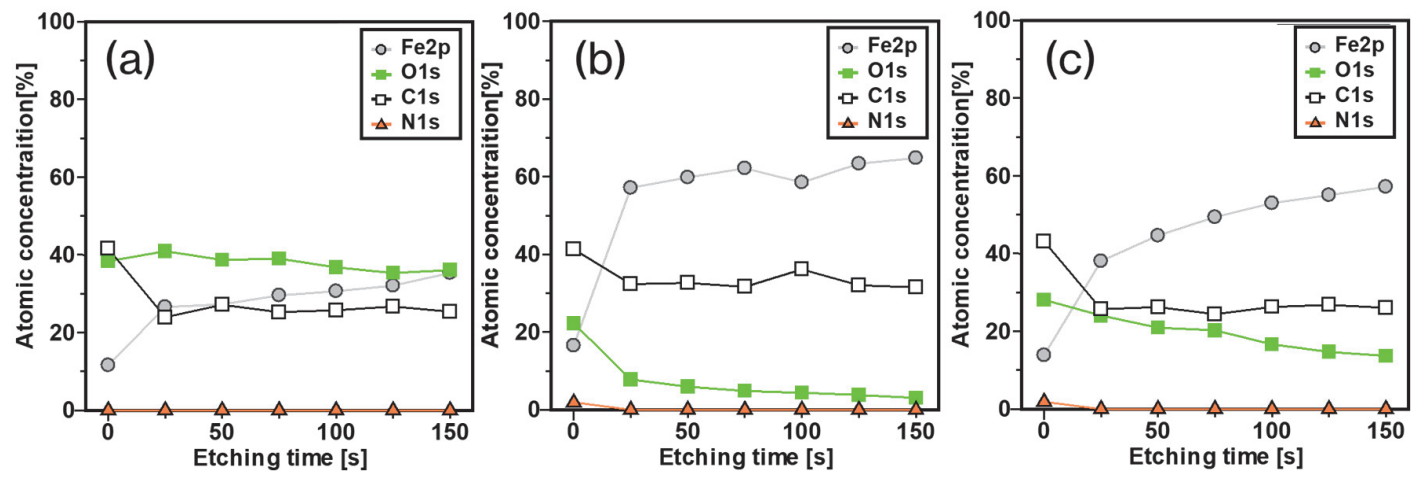

Fig. 12 Fe 2p, O 1s, C 1s, and N 1s XPS depth profiles of worn surfaces with different lubricants as functions of eating time. (a) DOS + ZDDP, (b) DOS + ZDDP + [BMPL][FAP], and (c) DOS + ZDDP + [BMIM][FAP].
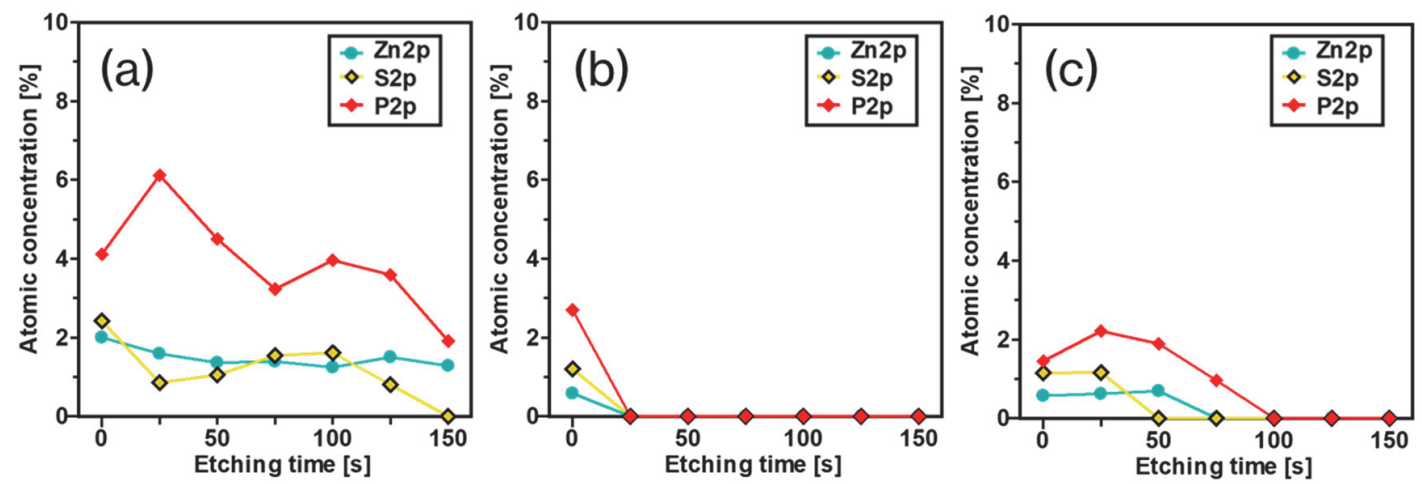

Fig. 13 Zn 2p, P 2p, and S 2p XPS depth profiles of worn surfaces with different lubricants as functions of the etching time. (a) DOS + ZDDP, (b) DOS + ZDDP + [BMPL][FAP], and (c) DOS + ZDDP + [BMIM][FAP]. 
$\mathrm{O}$ 1s and $\mathrm{C} 1 \mathrm{~s}$ decreases. This shows that the oxide layer and tribofilms were removed by Ar etching. $\mathrm{Zn}, \mathrm{S}$, and $\mathrm{P}$, which were derived from ZDDP, were detected in the depth profile obtained with DOS + ZDDP (Fig. 13). The ZDDP is widely known to form iron/zinc polyphosphates $[7,8,13]$. P $2 p$ peaks of DOS + ZDDP, DOS + ZDDP + [BMPL][FAP], and DOS + ZDDP + [BMIM][FAP] are placed at 133.5, 133.8, and 133.9 $\mathrm{eV}$ (Fig. 14), respectively. This result illustrates that a ZDDP tribofilm is formed on the sliding surface since these peaks are attributed to metal phosphates [58, 59]. In the case of DOS + ZDDP + IL solutions, the tribofilm thickness varied depending on the type of IL used. When DOS + ZDDP + [BMPL][FAP] was used, the concentration of $\mathrm{Zn}, \mathrm{S}$, and $\mathrm{P}$ decreased dramatically after an etching time of $25 \mathrm{~s}$, whereas in the case of DOS + ZDDP + [BMIM][FAP], they were detected up to 75,25 , and $50 \mathrm{~s}$, respectively. This observation suggests that the thickness of the ZDDP-derived tribofilm obtained with DOS + ZDDP + [BMPL] $[\mathrm{FAP}]$ was lower than that of the tribofilm obtained with DOS + ZDDP + [BMIM][FAP].

Figure 15 shows the F 1s spectra obtained with DOS + ZDDP $+[\mathrm{BMPL}][\mathrm{FAP}]$ and DOS + ZDDP $+[$ BMIM] $[\mathrm{FAP}]$ at an etching time of $0 \mathrm{~s}$. The results indicate that the ZDDP + IL mixed tribofilms were composed of $C-F_{x}$ bonds and iron fluoride at the outermost surface. In the case of DOS + ZDDP + [BMPL][FAP], the $F$ 1s spectrum exhibited four peaks at $682.9,684.9,686.6$, and $688.0 \mathrm{eV}$. The peaks at $684.9,686.6$, and $688.0 \mathrm{eV}$ are assigned to iron fluoride, $\mathrm{C}-\mathrm{F}_{3}$ bond, and $\mathrm{C}-\mathrm{F}_{2}$ bonds $[56,57]$, respectively. In the case of DOS + ZDDP + [BMIM] [FAP], peaks were observed at $682.6,684.7,686.8$, and $688.1 \mathrm{eV}$. The peaks at 684.7, 686.8 , and $688.1 \mathrm{eV}$ are also assigned to iron fluoride, $\mathrm{C}-\mathrm{F}_{3}$ bond and $\mathrm{C}^{-\mathrm{F}_{2}}$ bond $[56,57]$, respectively. The chemical compounds responsible for the peaks at 682.9 and $682.6 \mathrm{eV}$ in DOS + ZDDP $+[$ BMPL] $[\mathrm{FAP}]$ and DOS + ZDDP + [BMIM][FAP], respectively, could not be identified. The peaks corresponding to $C-\mathrm{F}_{x}$ bonds in these spectra suggest the existence of pure ILs. This result corroborates with ToF-SIMS observations. Meanwhile, iron fluoride was detected only by XPS. Therefore, it can be assumed that the ILs also formed iron fluoride tribofilms on the sliding surfaces. Finally, it can be inferred that both non-reacted and reacted IL existed on the sliding surfaces. Figure 16 maps the abundance of $\mathrm{F} 1 \mathrm{~s}$ (iron fluoride and $\mathrm{C}-\mathrm{F}_{\mathrm{x}}$ bonds) as functions of the etching time, respectively. $C-F_{x}$ bonds could be detected only at $0 \mathrm{~s}$, whereas iron fluoride could be detected from 0 to $150 \mathrm{~s}$. These results indicate that pure ILs were present only at the outermost surface and iron fluoride formed thicker films underneath it.

In summary, the ZDDP + [BMPL][FAP] tribofilm was thinner and was composed of a single ZDDP layer, while the DOS + ZDDP + [BMIM][FAP] tribofilm was comprised of three distinct layers, viz. a top layer consisting of pure [BMIM][FAP], middle layer with a ZDDP-derived tribofilm, and a bottom layer containing iron fluoride.
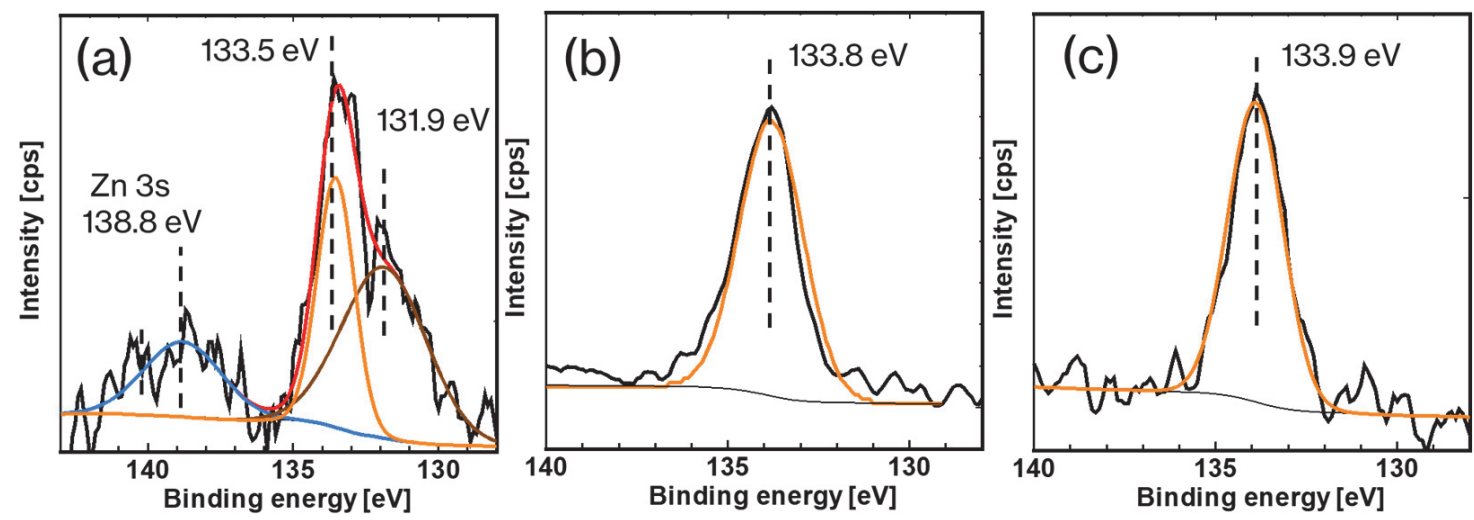

Fig. 14 P 2p peak on worn surface lubricated by (a) DOS + ZDDP, (b) DOS + ZDDP + [BMPL][FAP], and (c) DOS + ZDDP + [BMIM][FAP]
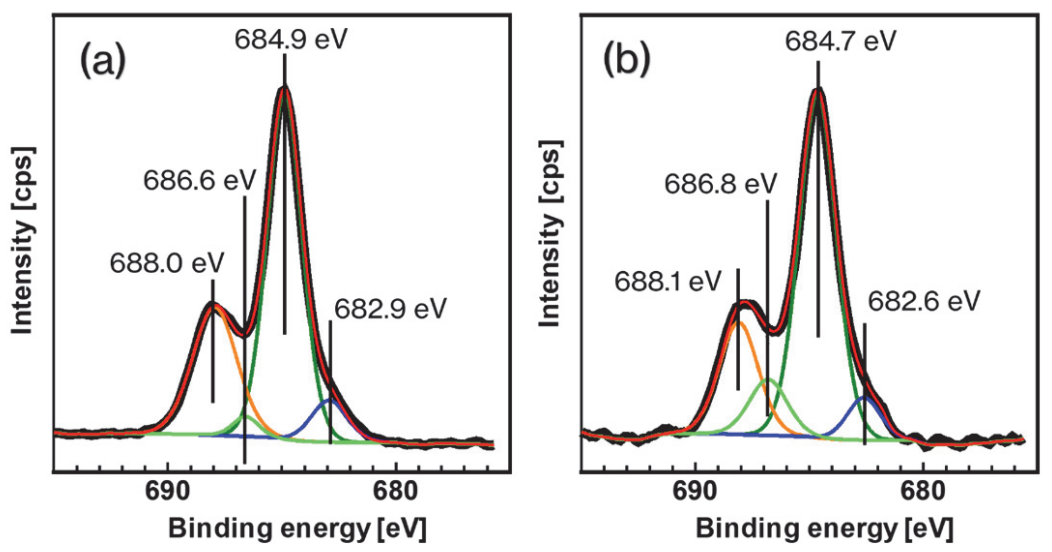

Fig. 15 XPS F 1s spectra of the wear tracks obtained with (a) DOS + ZDDP + [BMPL][FAP], and (b) DOS + ZDDP + [BMIM][FAP] 

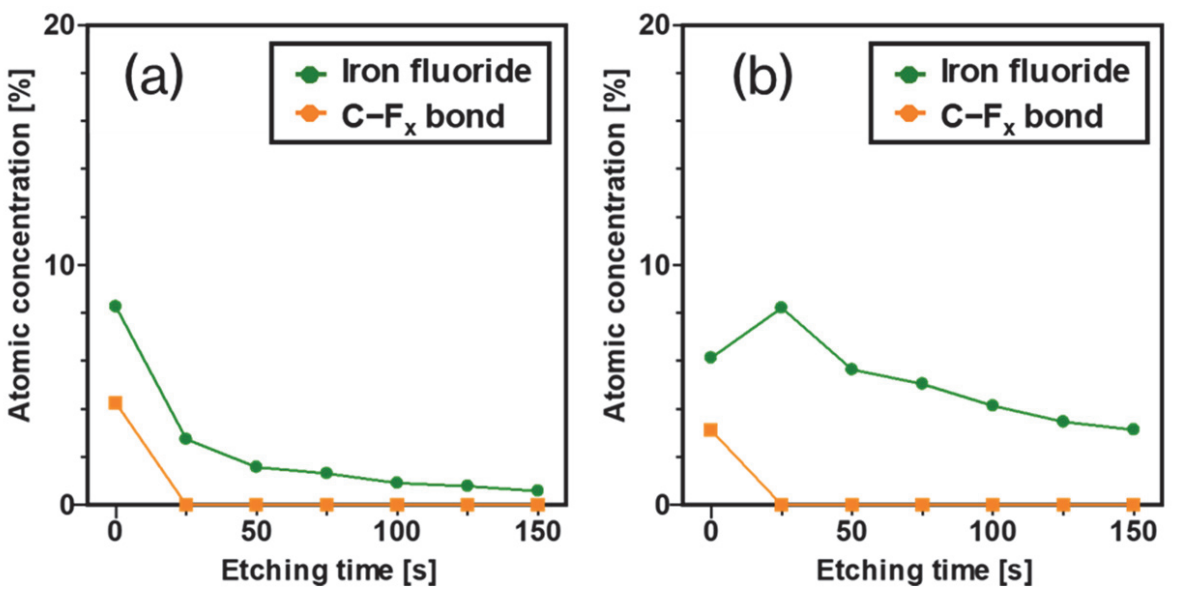

Fig. 16 F 1s XPS depth profiles of worn surfaces with different lubricants as functions of the etching time. (a) DOS + ZDDP + [BMPL][FAP], and (b) DOS + ZDDP + [BMIM][FAP].

\section{Discussion}

4.1 Tribofilm models formed by ZDDP + IL mixed solutions

Figure 17 shows a schematic depicting the reaction by which the DOS + ZDDP + [BMIM][FAP] system forms a tribofilm on the sliding surface. Based on the results in Figs. 15 and 16, we propose that these tribofilms were formed through three reactions with iron fluoride being formed in the initial sliding process (Fig. 17(b)), followed by the formation of a ZDDP tribofilm (Fig. 17(c)) and finally, the formation of an IL layer covering the ZDDP and iron fluoride tribofilms (Fig. 17(d)). As described in Section 3, during XPS analysis, iron fluoride was detected at the bottom of the tribofilm, indicating that the IL (containing fluorine) in the lubricant was the first component to undergo reaction with the steel surface (Fig. 17(b)). Note that if ZDDP is the first to react, the bottom layer should be composed of a sulfate/phosphate-based tribofilm.

The XPS results also showed that ZDDP-derived components were detected in the middle part of the tribofilm, which exhibited a pad-like structure (Fig. 7). Based on these results, it is suggested that the chemical reaction between ZDDP and the steel surface occurred after the formation of iron fluoride, and a pad-like ZDDP tribofilm was formed on top of the iron fluoride layer (Fig. 17(c)). ToF-SIMS and XPS analyses indicate that ILs were the main components in the outermost surface of the tribofilm; meanwhile, AFM confirmed the presence of a highly viscous film on the outermost surface. Together, these results suggest that the viscous IL layer covered the ZDDP and iron fluoride tribofilms. Based on this information, the tribo-process can be explained as follows. After the growth of the iron fluoride and ZDDP tribofilms, the triboreaction between ZDDP and the steel substrate slowed down as an increase in the tribofilm content on the steel surface resulted in a smaller exposed area of the steel surface. Consequently, non-reacted IL was adsorbed on the tribofilm composed of iron fluoride and ZDDP, which resulted in a highly viscous layer (Fig. 17(d)).

A schematic depicting the reaction between DOS + ZDDP
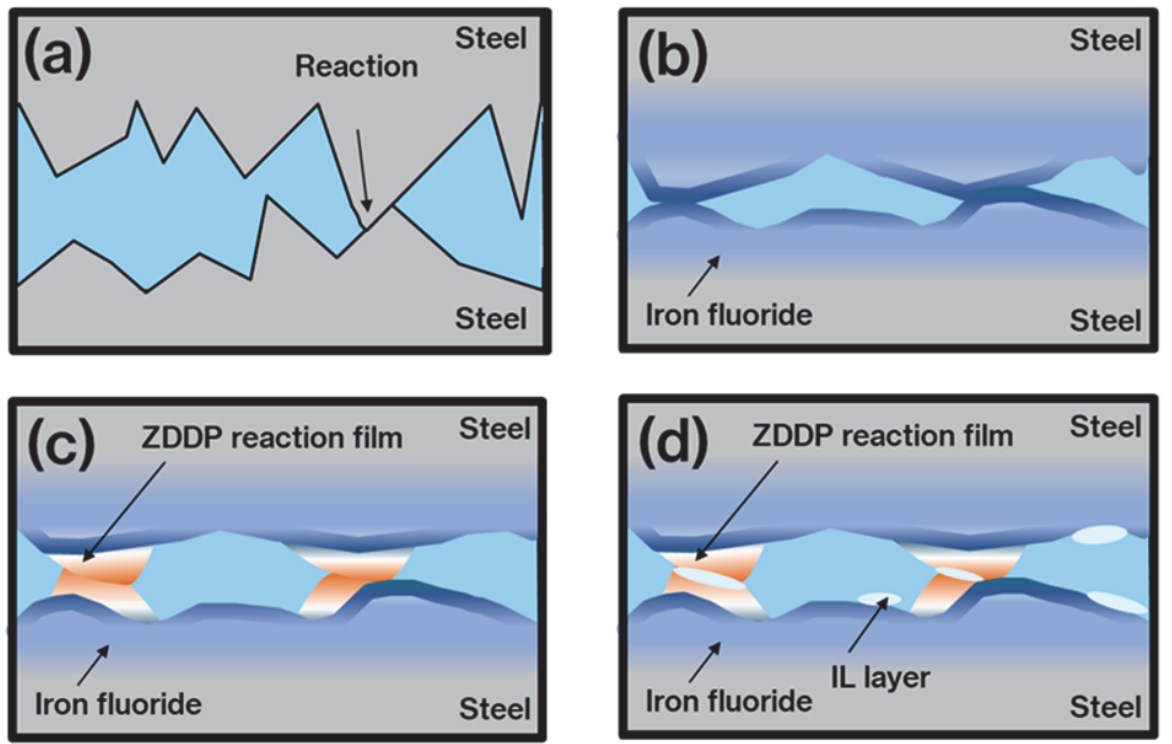

Fig. 17 Schematic of the reaction between DOS + ZDDP + [BMIM][FAP] and the steel surface. (a) Before tribo-reaction, (b) in the initial sliding phase, (c) after the formation of a ZDDP reaction film, and (d) after reaching stable friction conditions. 
$+[$ BMPL] $]$ FAP $]$ and the steel surface is shown in Fig. 18 When compared to the lubricant containing [BMIM][FAP], in this case, the formation of the ZDDP tribofilm followed a different process (Fig. 18(c)). The results of XPS (Fig. 13) analyses indicated a thinner ZDDP tribofilm with [BMPL][FAP] than with [BMIM][FAP]. In other words, the type of cation in the IL greatly affected the formation and growth of ZDDP tribofilms and this difference is expected to significantly affect the friction and anti-wear performance of the lubricants.

\subsection{Effect of cation type on tribofilm formation}

In this section, we shall discuss the effect of IL cation type on tribofilm formation. In this study, we hypothesized that the difference in the thickness of the ZDDP layer between the $\mathrm{DOS}+\mathrm{ZDDP}+[\mathrm{BMPL}][\mathrm{FAP}]$ and $\mathrm{DOS}+\mathrm{ZDDP}+[\mathrm{BMIM}][\mathrm{FAP}]$ systems can be attributed to their different adsorption behavior instead of the decomposition temperature.

It is known that the decomposition temperature largely contributes to the formation of tribofilms [7, 22, 60]. Figure 5 shows that both [BMPL][FAP] and [BMIM][FAP] exhibited similar decomposition temperatures in the range of $340-350^{\circ} \mathrm{C}$, while ZDDP decomposed in the range of $100-200^{\circ} \mathrm{C}[7,8,21]$. These temperatures suggest that the reaction temperature of ZDDP is lower than that of IL on the sliding surfaces. Therefore, ZDDP can react more readily with the sliding surface when compared to [BMIM][FAP] and [BMPL][FAP]. However, XPS depth analysis indicated the formation of iron fluoride, which is a product of the reaction between the IL and steel substrate; this, in turn, indicated that the steel substrate reacted preferentially with the IL rather than ZDDP. Mori et al. reported that the catalysis effects by generating the nascent surface during friction cause the decomposition of ILs [60], and ILs form the tribofilm at temperature by far lower than onset at TGA. Therefore, it is considered that IL decomposes at low temperatures influenced by the generation of the nascent surface during friction, even when the ionic liquid is used as additives. Also, we used the same anion IL in our experiments. Therefore, catalysis effects of the nascent on tribofilm formation are considered to be similar between [BMPL][FAP] and [BMIM]
[FAP]. Therefore, it might be inferred that the decomposition temperature of each IL is not the driving factor for the tribofilm formation of ZDDP. During ZDDP tribofilm formation, ZDDP molecules firstly adsorb on the sliding surface and subsequently react with the sliding surfaces [7]. Miklozic et al. reported that friction modifiers, added to the base oil together with ZDDP, suppressed the formation of ZDDP tribofilms by blocking the adsorption of ZDDP on the surface [61]. Khanmohammadi et al. also reported that the [BMPL][FAP], which has faster adsorption kinetic of IL compared to other two ILs ((2-hydroxyethyl) trimethylammonium dimethylphosphate and Tributylmethylphosphonium dimethylphosphate), shows superior friction and anti-wear properties, and it is suggested that the F element, which is composed of [FAP] anion, causes the faster adsorption thus low friction [62].

Based on these reports, we hypothesized that ILs were first adsorbed on the steel substrate, which favors the reaction between ILs and the steel substrate; this reaction subsequently resulted in the formation of iron fluoride by the existence of nascent surface while the ILs have high thermal stability. Therefore, the adsorption properties of the ILs and ZDDP exerted a significant effect on the initial tribofilm formation rather than the decomposition temperature. The subsequent tribofilm formation by ZDDP was also affected by the adsorption properties of ILs on the iron fluoride layer because the growth of ZDDP tribofilms on the iron fluoride surface is strongly dependent on the type of IL.

\subsection{Friction mechanism of different lubricant systems}

When DOS + ZDDP + [BMIM] [FAP] was used as the lubricant, a lower friction coefficient was observed when compared to the case in which DOS + ZDDP + [BMPL][FAP] was used; this difference may be attributed to the thicker middle layer of ZDDP in the tribofilm produced by the former. It is generally known that ZDDP tribofilms increase friction on the steel substrates when using poly-alpha-olefins and mineral oil as base oil $[7,61,63,64]$. However, based on the results described in Section 3, we hypothesize that the ZDDP tribofilm reduced friction. In fact, DOS + ZDDP exhibited a lower friction
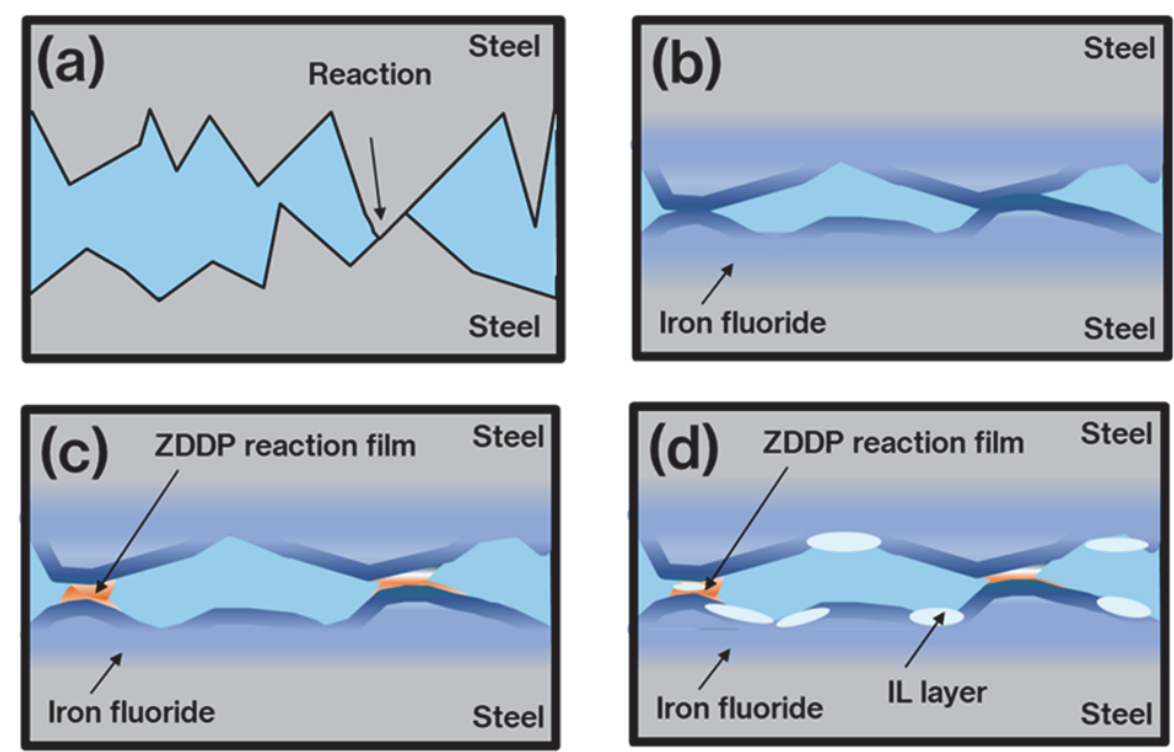

Fig. 18 Schematic of the reaction between DOS + ZDDP + [BMPL][FAP] and the steel surface. (a) Before tribo-reaction, (b) in the initial sliding phase, (c) after the formation of a ZDDP reaction film, and (d) after reaching stable friction conditions. 
coefficient than pure DOS. Guo et al. reported that ZDDP reduces friction by using ester base oil diisooctylsebacate (DIOS) [65]. Hence, we believe that ZDDP-derived tribofilms reduce friction when used in combination with DOS, an ester-based oil. Meanwhile, the friction coefficient observed with DOS + ZDDP + [BMIM][FAP] was smaller than that obtained with DOS + ZDDP; meanwhile, the latter resulted in a thicker ZDDP tribofilm. Therefore, the presence of an IL in the outermost layer and iron fluoride also contribute to friction reduction. The AFM results shown in Fig. 8 indicate that the outermost IL layer is viscous. However, no viscous layers were detected on surfaces with DOS +ZDDP as the lubricant. Therefore, it may be surmised that viscous IL layers decrease friction by reducing adhesion through direct contact between the ZDDP-derived film and iron fluoride. Regarding the effect of cations, DOS + ZDDP $+[$ BMPL] $]$ FAP $]$ resulted in a higher friction coefficient than DOS + ZDDP + [BMIM][FAP]. This is because thinner ZDDP tribofilms are less defensive for direct contact, although they form a highly viscous layer. These results also suggest that a balance between ZDDP formation and the IL layer is important to reduce friction.

\subsection{Anti-wear mechanism of different lubricant systems}

As shown in Fig. 3, ZDDP and IL-containing lubricants resulted in a significant wear reduction. Mourhatch et al. reported that fluorinated ZDDP exhibits significant anti-wear properties by producing iron fluoride, which suggests that iron fluoride enhances the anti-wear characteristics of a substrate when ZDDP is combined with F. In our tribofilm model, in the case of DOS + ZDDP + ILs (Figs. 17 and 18), the bottom layer contains iron fluoride $[64,66]$. This implies that chemical reactions occur readily between fluorine and the steel surface, resulting in a reduction in initial wear. In addition, DOS + ZDDP $+[$ BMIM] $[$ FAP] showed the lowest wear volume, probably due to the presence of a thick ZDDP layer in the tribofilm.

\section{Conclusions}

In summary, the synergism in the tribological properties of ZDDP and ILs as lubricant additives was investigated by changing the chemical composition of the IL used. Surface analysis was conducted to examine the effect of IL structure on the frictional and anti-wear properties of lubricated steel surfaces and tribofilm formation. The major conclusions were as follows:

(1) The combination of ZDDP and [BMIM][FAP] with the base oil resulted in a very low friction coefficient and wear volume. In addition, the mixed tribofilm thus produced improved the friction and anti-wear properties of the substrates.

(2) ZDDP and IL-mixed tribofilms consisted of a viscous pure IL layer on top of the ZDDP tribofilm, which improved the friction and anti-wear characteristics of the substrates under boundary lubrication conditions.

(3) The chemical composition and thickness of tribofilms can be influenced by changing the cation component in the ILs used as lubricant additives together with ZDDP. The chemical composition of the resultant mixed tribofilms is a key factor in achieving both lower friction and wear.

\section{References}

[1] Holmberg, K. and Erdemir, A., “The Impact of Tribology on Energy
Use and $\mathrm{CO}_{2}$ Emission Globally and in Combustion Engine and Electric Cars," Tribology International, 135, 2019, 389-396.

[2] Thangarasu, V., Balaji, B. and Ramanathan, A., "Experimental Investigation of Tribo-Corrosion and Engine Characteristics of Aegle Marmelos Correa Biodiesel and Its Diesel Blends on Direct Injection Diesel Engine," Energy, 171, 2019, 879-892.

[3] Howell-Smith, S., Rahnejat, H., King, P. D. and Dowson, D., "Reducing in-Cylinder Parasitic Losses Through Surface Modification and Coating," Proceedings of the Institution of Mechanical Engineers, Part D: Journal of Automobile Engineering, 228, 2014, 391-402.

[4] Maruyama, M. and Gill, N. Y., "Trends of Fuel-Economy/LowViscosity Gasoline Engine Oils," Journal of Japanese Society of Tribologists, 59, 2014, 380-386 (in Japanese).

[5] Maroto-Centeno, J. A., Pérez-Gutiérrez, T., Fernández-RuízMorón, L. and Quesada-Pérez, M., "Prediction of Fuel Economy Performance of Engine Lubricants Based on Laboratory Bench Tests," Tribology International, 94, 2016, 67-70.

[6] Cui, J., Oberoi, S., Briggs, S. and Goldmints, I., "A Viscosity Modifier Solution to Reconcile Fuel Economy and Durability in Diesel Engines," Tribology International, 101, 2016, 43-48.

[7] Spikes, H., "The History and Mechanisms of ZDDP," Tribology Letters, 17, 2004, 469-489.

[8] Zhang, J. and Spikes, H., "On the Mechanism of ZDDP Antiwear Film Formation," Tribology Letters, 63, 2016, 24.

[9] Habeeb, J. J. and Stover, W. H., "The Role of Hydroperoxides in Engine Wear and the Effect of Zinc Dialkyldithiophosphates," ASLE Transactions, 30, 1986, 419-426.

[10] Rounds, F., "Effects of Hydroperoxides on Wear as Measured in Four-Ball Wear Tests," Tribology Transactions, 36, 1993, 297-303.

[11] Belin, M., Martin, J. M. and Mansot, J. L., "Role of Iron in the Amorphization Process in Friction-Induced Phosphate Glasses," Tribology Transactions, 32, 1989, 410-413.

[12] Willermet, P. A., Dailey, D. P., Carter, R. O., Schmitz, P. J. and Zhu, W., "Mechanism of Formation of Antiwear Films from Zinc Dialkyldithiophosphates," Tribology International, 28, 1995, 177187.

[13] Gosvami, N. N., Bares, J. A., Mangolini, F., Konicek, A. R., Yablon, D. G. and Carpick, R. W., "Mechanisms of Antiwear Tribofilm Growth Revealed In Situ by Single-Asperity Sliding Contacts," Science, 348, 2015, 102-106.

[14] Kontou, A., Taylor, R. I. and Spikes, H. A., "Effects of Dispersant and ZDDP Additives on Fretting Wear," Tribology Letters, 69, 2021, 6.

[15] Shimizu, Y. and Spikes, H. A., "The Tribofilm Formation of ZDDP under Reciprocating Pure Sliding Conditions," Tribology Letters, 64, 2016, 46.

[16] Sieber, I., Meyer, K., Kloss, H. and Schöpke, A., “Characterization of Boundary Layers Formed by Different Metal Dithiophosphates in a Four-Ball Machine," Wear, 85, 1983, 43-56.

[17] Dawczyk, J., Morgan, N., Russo, J. and Spikes, H., "Film Thickness and Friction of ZDDP Tribofilms," Tribology Letters, 67, 2019, 34.

[18] Qu, J., Bansal, D. G., Yu, B., Howe, J. Y., Luo, H., Dai, S., Li, H., Blau, P. J., Bunting, B. G., Mordukhovinch, G. and Smolenski, D. J., "Antiwear Performance and Mechanism of an Oil-Miscible Ionic Liquid as a Lubricant Additive," ACS Applied Materials \& Interfaces, 4, 2012, 997-1002.

[19] Qu, J., Truhan, J. J., Dai, S., Luo, H. and Blau, P. J., “Ionic Liquids with Ammonium Cations as Lubricants or Additives," Tribology Letters, 22, 2006, 207-214.

[20] Zhou, Y. and Qu, J., "Ionic Liquids as Lubricant Additives: A Review," ACS Applied Materials \& Interfaces, 9, 2017, 3209-3222.

[21] Minami, I., “Ionic Liquids in Tribology," Molecules, 14, 2009, 2286- 
2305

[22] Kawada, S., Watanabe, S., Tadokoro, C., Tsuboi, R. and Sasaki, S., "Lubricating Mechanism of Cyano-Based Ionic Liquids on Nascent Steel Surface," Tribology International, 119, 2018, 474-480.

[23] Minami, I. and Mori, S., "Tribology of Ionic Liquids," Journal of the Surface Science Society of Japan, 28, 2007, 311-317 (in Japanese).

[24] Sanes, J., Avilés, M. D., Saurín, N., Espinosa, T., Carrión, F. J. and Bermúdez, M. D., "Synergy between Graphene and Ionic Liquid Lubricant Additives," Tribology International, 116, 2017, 371-382.

[25] Qu, J., Luo, H., Chi, M., Ma, C., Blau, P. J., Dai, S. and Viola, M. B., "Comparison of an Oil-Miscible Ionic Liquid and ZDDP as a Lubricant Anti-Wear Additive," Tribology International, 71, 2014, 88-97.

[26] Kondo, H., Ito, M., Hatsuda, K., Yun, K. and Watanabe, M., “Novel Ionic Lubricants for Magnetic Thin Film Media," IEEE Transactions on Magnetics, 49, 2013, 3756-3759.

[27] Kondo, Y., Yagi, S., Koyama, T., Tsuboi, R. and Sasaki, S., "Lubricity and Corrosiveness of Ionic Liquids for Steel-on-Steel Sliding Contacts," Proceedings of the Institution of Mechanical Engineers, Part J: Journal of Engineering Tribology, 226, 2012, 991-1006.

[28] Watanabe, S., Takiwatari, K., Nakano, M., Miyake, K., Tsuboi, R. and Sasaki, S., "Molecular Behavior of Room-Temperature Ionic Liquids under Lubricating Condition," Tribology Letters, 51, 2013, 227-234.

[29] Yang, S., Zhang, D., Wong, J. S. S. and Cai, M., "Interactions between ZDDP and an Oil-Soluble Ionic Liquid Additive," Tribology International, 158, 2021, 106938

[30] Cai, M., Liang, Y., Yao, M., Xia, Y., Zhou, F. and Liu, W., "Imidazolium Ionic Liquids as Antiwear and Antioxidant Additive in Poly(ethylene glycol) for Steel/Steel Contacts," ACS Applied Materials \& Interfaces, 2, 2010, 870-876.

[31] Cai, M. R., Yu, Q. L., Liu, W. M. and Zhou, F., "Ionic Liquid Lubricants: When Chemistry Meets Tribology," Chemical Society Reviews, 2020, 7753-7818.

[32] Guo, W., Zhou, Y., Sang, X.H., Leonard, D.N., Qu, J., Poplawsky, J.D "Atom probe tomography unveils formation mechanisms of wearprotective tribofilms by ZDDP, ionic liquid, and their combination," ACS Applied Materials \& Interfaces, 9, 2017, 23152-23163.

[33] Qu, J., Barnhill, W. C., Luo, H., Meyer, H. M., Leonard, D. N., Landauer, A. K., Kheireddin, B., Gao, H., Papke, B. L. and Dai, S., "Synergistic Effects between Phosphonium-Alkylphosphate Ionic Liquids and Zinc Dialkyldithiophosphate (ZDDP) as Lubricant Additives," Advanced Materials, 27, 2015, 4767-4774.

[34] Ogunniyi, D.S., "Castor Oil: A Vital Industrial Raw Material," Bioresource Technology, 97, 2006, 1086-1091.

[35] Xu, Y., Hu, E., Hu, K., Xu, Y. and Hu, X., "Formation of an Adsorption Film of $\mathrm{MoS}_{2}$ Nanoparticles and Dioctyl Sebacate on a Steel Surface for Alleviating Friction and Wear," Tribology International, 92, 2015, 172-183.

[36] Puscas, C., Bandur, G., Modra, D. and Nutiu, R., “Mixtures of Vegetable Oils and Di-2-ethylhexyl-sebacate as Lubricants," Journal of Synthetic Lubrication, 23, 2006, 185-196.

[37] Jiménez, A. E. and Bermúdez, M. D., “Imidazolium Ionic Liquids as Additives of the Synthetic Ester Propylene Glycol Dioleate in Aluminium-Steel Lubrication," Wear, 265, 2008, 787-798.

[38] Tung, S. C. and McMillan, M. L., “Automotive Tribology Overview of Current Advances and Challenges for the Future," Tribology International, 37, 2004, 517-536.

[39] Ye, J., "Mechanism of Friction Reduction and Nanoscale Controlling Factors in MoDTC/ZDDP Tribofilms," Journal of Japanese Society of Tribologists, 51, 2006, 627-632 (in Japanese).

[40] Kalin, M., Oblak, E. and Akbari, S., "Evolution of the Nano-Scale Mechanical Properties of Tribofilms Formed from Low- and HighSAPS Oils and ZDDP on DLC Coatings and Steel," Tribology
International, 96, 2016, 43-56.

[41] Greenall, A., Neville, A., Morina, A. and Sutton, M., “Investigation of the Interactions between a Novel, Organic Anti-Wear Additive, ZDDP and Overbased Calcium Sulphonate," Tribology International, 46, 2012, 52-61.

[42] Young, T. J., Crocker, L. E., Broughton, W. R., Ogin, S. L. and Smith, P. A., "Observations on Interphase Characterisation in Polymer Composites by Nano-Scale Indentation Using AFM and FEA," Composites Part A: Applied Science and Manufacturing, 50, 2013, $39-43$.

[43] Lonnroth, N., Muhlstein, C. L., Pantano, C. and Yue, Y., "Nanoindentation of Glass Wool Fibers," Journal of Non-Crystalline Solids, 354, 2008, 3887-3895.

[44] Khanna, S. K., Ranganathan, P., Yedla, S. B., Winter, R. M. and Paruchuri, K., "Investigation of Nanomechanical Properties of the Interphase in a Glass Fiber Reinforced Polyester Composite Using Nanoindentation," Journal of Engineering Materials and Technology, 125, 2003, 90-96.

[45] Nalam, P. C., Gosvami, N. N., Caporizzo, M. A., Composto, R. J. and Carpick, R. W., “Nano-Rheology of Hydrogels Using Direct Drive Force Modulation Atomic Force Microscopy," Soft Matter, 11, 2015, 8165-8178.

[46] Qian, L. and Zhao, H., "Nanoindentation of Soft Biological Materials," Micromachines, 9, 2018, 654.

[47] Okubo, H., Watanabe, S., Tadokoro, C. and Sasaki, S., "Effects of Structure of Zinc Dialkyldithiophosphates on Tribological Properties of Tetrahedral Amorphous Carbon Film under Boundary Lubrication," Tribology International, 98, 2016, 26-40.

[48] Okubo, H., Watanabe, S., Tadokoro, C. and Sasaki, S., "Effects of Concentration of Zinc Dialkyldithiophosphate on the Tribological Properties of Tetrahedral Amorphous Carbon Films in Presence of Organic Friction Modifiers," Tribology International, 94, 2016, 446457.

[49] Hofmann, S., "Surface Segregation during Sputtering at Elevated Temperatures," Materials Science and Engineering, 42, 1980, 55-58.

[50] Hashimoto, S., Tanaka, A., Murata, A. and Sakurada, T., "Formulation for XPS Spectral Change of Oxides by Ion Bombardment as a Function of Sputtering Time," Surface Science, $556,2004,22-32$

[51] Malherbe, J. B., Hofmann, S. and Sanz, J. M., “Preferential Sputtering of Oxides: A Comparison of Model Predictions with Experimental Data," Applied Surface Science, 27, 1986, 355-365.

[52] Zhang, Y., Cai, T., Shang, W., Sun, L., Liu, D., Tong, D. and Liu, S., "Environmental Friendly Polyisobutylene-Based Ionic Liquid Containing Chelated Orthoborate as Lubricant Additive: Synthesis, Tribological Properties and Synergistic Interactions with ZDDP in Hydrocarbon Oils," Tribology International, 115, 2017, 297-306.

[53] Spadaro, F., Rossi, A., Laine, E., Woodward, P. and Spencer, N. D., "Elucidating the Resistance to Failure under Tribological Tests of Various Boron-Based Films by XPS and ToF-SIMS," Applied Surface Science, 425, 2017, 948-964.

[54] Minfray, C., Martin, J. M., De Barros, M. I., Le Mogne, T., Kersting, R. and Hagenhoff, B., "Chemistry of ZDDP Tribofilm by ToF-SIMS," Tribology Letters, 17, 2004, 351-357.

[55] Kawada, S., Watanabe, S., Tsuboi, R., Sasaki, S. and Prakash, B., "Lubrication Mechanism of Halogen-Free Ionic Liquids," Tribology Online, 12, 2017, 155-161.

[56] Hsu, H. L., Leong, K. R., Teng, I. J., Halamicek, M., Juang, J. Y., Jian, S. R., Qian, L. and Kherani, N. P., "Reduction of Photoluminescence Quenching by Deuteration of Ytterbium-Doped Amorphous Carbon-Based Photonic Materials," Materials, 7, 2014, 5643-5663.

[57] Wen, Z., Zhu, L., Li, L., Sun, L., Cai, H. and Ye, Z., "A FluorineMediated Hydrothermal Method to Synthesize Mesoporous 
Rhombic ZnO Nanorod Arrays and Their Gas Sensor Application," Dalton Transactions, 42, 2013, 15551-15554.

[58] Morina, A., Neville, A., Priest, M. and Green, J. H., “ZDDP and MoDTC Interactions and Their Effect on Tribological Performance Tribofilm Characteristics and Its Evolution," Tribology Letters, 24, 2006, 243-256.

[59] Barbaux, Y., Dekiouk, M., Le Maguer, D., Gengembre, L., Huchette, D. and Grimblot, J., "Bulk and Surface Analysis of a Fe-P-O Oxydehydrogenation Catalyst," Applied Catalysis A: General, 90, 1992, 51-60.

[60] Lu, R., Mori, S., Kobayashi, K. and Nanao, H., "Study of Tribochemical Decomposition of Ionic Liquids on a Nascent Steel Surface," Applied Surface Science, 255, 2009, 8965-8971.

[61] Muraki, M. and Oshima, A., "Influence of Alkyl Group on Friction-Velocity Characteristics of Primary-Alkyl Zinc Dialkyldithiophosphate," Journal of Japanese Society of Tribologists, 57, 2012, 417-425 (in Japanese).

[62] Khanmohammadi, H., Wijanarko, W. and Espallargas, N., "Ionic Liquids as Additives in Water-Based Lubricants: From Surface
Adsorption to Tribofilm Formation," Tribology Letters, 68, 2020, 130.

[63] Miklozic, K. T., Forbus, T. R. and Spikes, H. A., "Performance of Friction Modifiers on ZDDP-Generated Surfaces," Tribology Transactions, 50, 2007, 328-335.

[64] Mourhatch, R. and Aswath, P. B., "Tribological behavior and Nature of Tribofilms Generated from Fluorinated ZDDP in Comparison to ZDDP under Extreme Pressure Conditions-Part 1: Structure and Chemistry of Tribofilms," Tribology International, 44, 2011, 187-200.

[65] Guo, Z., Zhang, Y., Wang, J., Gao, C., Zhang, S., Zhang, P. and Zhang, Z., "Interactions of $\mathrm{Cu}$ Nanoparticles with Conventional Lubricant Additives on Tribological Performance and Some Physicochemical Properties of an Ester Base Oil," Tribology International, 141, 2020, 105941.

[66] Mourhatch, R. and Aswath, P. B., "Tribological behavior and Nature of Tribofilms Generated from Fluorinated ZDDP in Comparison to ZDDP under Extreme Pressure Conditions-Part II: Morphology and Nanoscale Properties of Tribofilms," Tribology International, 44, 2011, 201-210.

This paper is licensed under the Creative Commons Attribution-NonCommercial-NoDerivatives 4.0 International (CC BYNC-ND 4.0) License. This allows users to copy and distribute the paper, only upon conditions that (i) users do not copy or distribute such paper for commercial purposes, (ii) users do not change, modify or edit such paper in any way, (iii) users give appropriate credit (with a link to the formal publication through the relevant DOI (Digital Object Identifier)) and provide a link to this license, and (iv) users acknowledge and agree that users and their use of such paper are not connected with, or sponsored, endorsed, or granted official status by the Licensor (i.e. Japanese Society of Tribologists). To view this license, go to https://creativecommons.org/licenses/by-nc-nd/4.0/. Be noted that the third-party materials in this article are not included in the Creative Commons license, if indicated on the material's credit line. The users must obtain the permission of the copyright holder and use the third-party materials in accordance with the rule specified by the copyright holder. 